\title{
Energy Efficient Control of the Dehumidification Process in Heat Exchangers with Air Bypass
}

\author{
Stephan Kusche ${ }^{1, *}$, and André Badura ${ }^{1}$ \\ ${ }^{1}$ HTW Berlin, Department of Engineering, 12459 Berlin, Germany
}

\begin{abstract}
This paper deals with an energy efficient approach for the dehumidification process of supply air. The basic concept consists of an air bypass, which separates the airstream. Later the unprocessed air is mixed with the conditioned air. This mixing allows one to avoid the energy consuming reheating of the air stream. Application of this concept demands for a sophisticated controller. In this case a state space controller is designed. Therefore, the underlying model for the heat exchanger is derived and a Krylov Space based reduction method is applied. This model is broadened for the bypass. The overall linear model is derived via numerical linearization.
\end{abstract}

\section{Nomenclature}

In this papers the following symbols

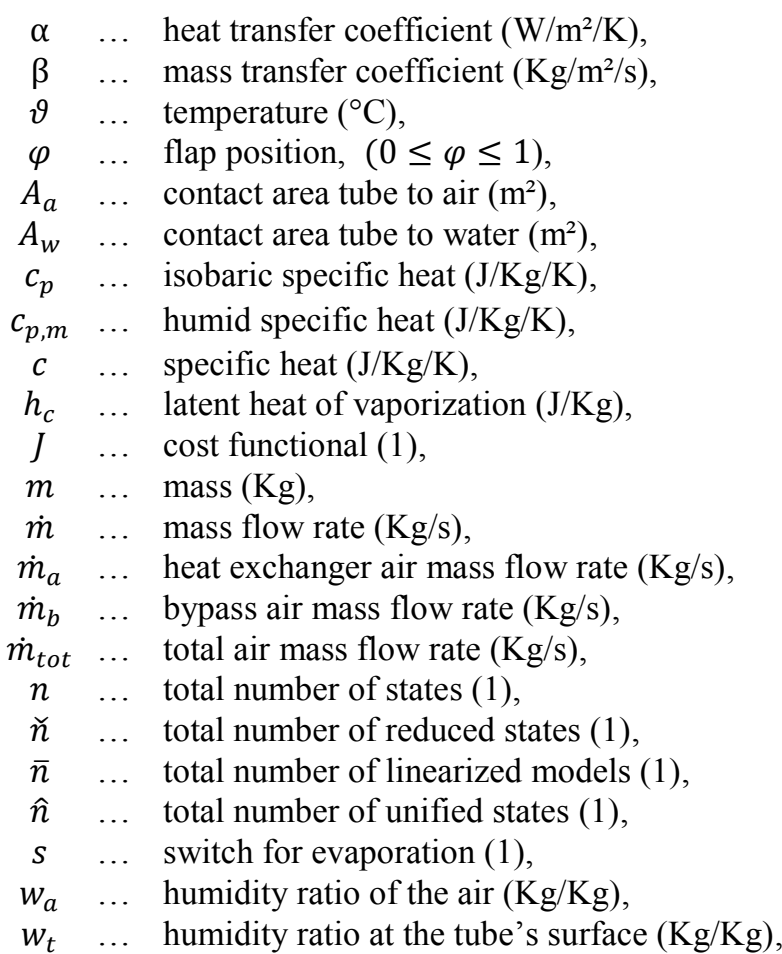

vectors

$\begin{array}{lll}\boldsymbol{a} \in \mathbb{R}^{n \times 1} & \ldots & \text { constant offset vector, } \\ \boldsymbol{b} \in \mathbb{R}^{n \times 1} & \ldots & \text { constant offset vector, } \\ \boldsymbol{u} \in \mathbb{R}^{5 \times 1} & \ldots & \text { input vector, } \\ \boldsymbol{x} \in \mathbb{R}^{n \times 1} & \ldots & \text { state vector, } \\ \boldsymbol{y} \in \mathbb{R}^{5 \times 1} & \ldots & \text { output vector, } \\ \boldsymbol{z} \in \mathbb{R}^{2 \times 1} & \ldots & \text { vector of controlled variables, }\end{array}$

and matrices

$$
\begin{array}{cll}
A \in \mathbb{R}^{n \times n} & \ldots & \text { state matrix, } \\
B \in \mathbb{R}^{n \times 5} & \ldots & \text { input matrix, } \\
C \in \mathbb{R}^{5 \times n} & \ldots & \text { output matrix, } \\
D \in \mathbb{R}^{5 \times 5} & \ldots & \text { feedthrough matrix, } \\
K \in \mathbb{R}^{2 \times \check{n}} & \ldots & \text { linear state space controller, } \\
U \in \mathbb{R}^{n \times n} & \ldots & \text { unitary matrix (SVD), } \\
M \in \mathbb{R}^{\hat{n} \times \check{n}} & \ldots & \text { projection matrix, } \\
N \in \mathbb{R}^{n \bar{n} \times n \bar{n}} & \ldots & \text { unitary matrix (SVD), } \\
Q_{y} \in \mathbb{R}^{2 \times 2} & \ldots & \text { weight matrix output variables, } \\
R \in \mathbb{R}^{n \times \hat{n}} & \ldots & \text { projection matrix, } \\
R_{u} \in \mathbb{R}^{2 \times 2} & \ldots & \text { weight matrix actuating variables, } \\
\Sigma \in \mathbb{R}^{n \times n \bar{n}} & \ldots & \text { diagonal matrix (SVD), } \\
V, W \in \mathbb{R}^{n \times \check{n}} & \ldots & \text { projection matrix }
\end{array}
$$

are used. They are accompanied by the following indices

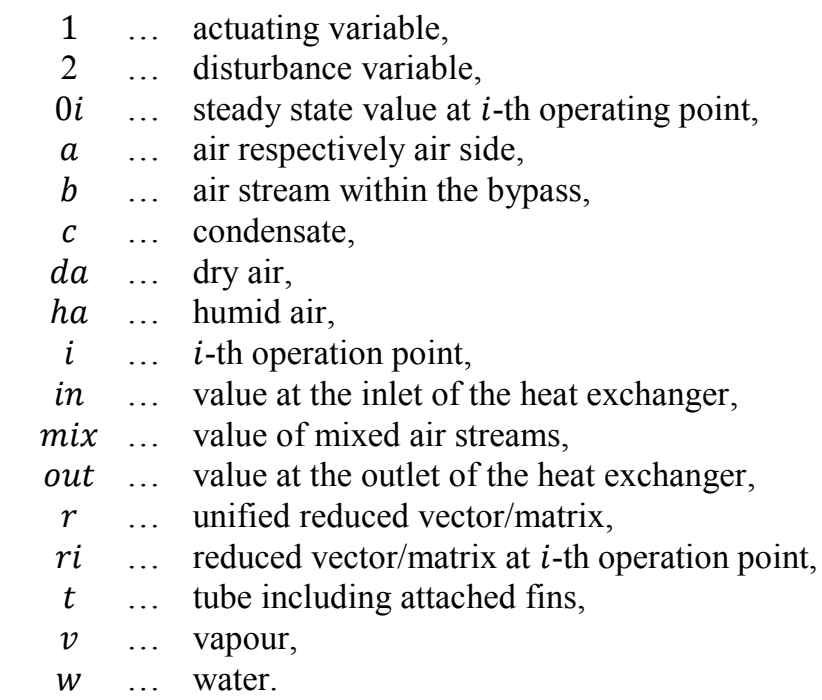

\footnotetext{
* Corresponding author: kusche@htw-berlin.de
} 


\section{Introduction}

The consumption of power rises worldwide. One not negligible division is the heating, ventilation and air conditioning (hvac) of buildings. The underlying reason for that is the trend towards urbanization and the global warming of the earth, especially in urban areas $[1,2]$. Due to increasing demands in standard of living (thermal wellbeing) and due to pure necessity (clean air heavily loaded from particulates), hvac units will be in great demand. The whole process of cleaning and conditioning air is very energy consumptive [3], which brings us to the starting point again. To break this vicious circle, one has to change human behaviour and thinking, which seems not to be very likely. As an alternative the process itself needs to be optimized. In this paper the following two approaches to achieve this objective will be discussed: Optimization in the process of cooling and dehumidification itself $[4,5]$ and development of an optimal control for this process $[6,7]$.

To optimize the process of cooling and dehumidification one can start with a standard hvac unit. Its task is to condition the supply air of a room in a way, that air temperature and air humidity lie within the range of comfort. For simplicities sake one can choose an explicit given reference point within this range. If the unconditioned air temperature is higher than that of the reference point, cooling is necessary. Additionally, if the unconditioned air humidity is higher than that of the reference point, dehumidification is necessary. Typically done by undercooling the air below the dew point and subsequent heating to meet the reference point's temperature. Without any further actions the energy spent into the reheating process is lost. Way out of this can be to use a heat pump between the two heat exchangers or to reheat the air from another source, for example the room exit air. In this paper the usage of an air bypass system shall be discussed. The principle of the air bypass is the following. A part of the air stream is separated before cooling by the heat exchanger. Afterwards the cooled air from the heat exchanger is mixed with the untreated air, with the result that the air temperature, as well as the air humidity, are rising. Reheating can be skipped under proper conditions which will be discussed later in detail. In order to obtain the air at the reference points conditions, a proper control structure has to be chosen. Therefore, a model of the heat exchanger together with the air bypass has been developed. This model is linearized and simplified using a Krylov subspace-based reduction method. From this linearized and simplified model, a state space controller will be developed.

\section{Model of the system}

The overall system consists of the heat exchanger and the air bypass. The first one is the more complex part and will be modelled separately. To get rid of the first models complexity in terms of the number of state space variables, it will be linearized and reduced. These steps can be found in section 2.1 while section 2.2 describes the extension by the air bypass. The resulting model is linear.

\subsection{Model of the heat exchanger}

The heat exchanger used, is a standard crossflow fin and tube heat exchanger manufactured by Hombach. The cooling fluid is usually water, optionally mixed with glycol for protection against freezing. Tubes are made of copper advanced by fins made of aluminium. In the modelling process the heat exchanger is divided into cells (2.1.1). In each cell energy balance equations are used (2.1.2). For further information about cell methods in heat exchangers the reader is referred to $[8,9]$. By connecting the cells according to the mass streams of air and water, the whole heat exchanger will be represented (2.1.3). The linearization and reduction process gives a simplified model suitable for further utilization (2.1.4).

\subsubsection{Subdivision into cells}

The common geometry of crossflow heat exchangers implies a discretisation along each tube. Let one cell consist of one tube element and let the attached fins be separated and uniquely assigned to each tube. This can be done by either hexagonal or rectangular shaped cells. The first ones are used if the tubes are placed staggered, the second ones if the tubes are placed in line to each other.

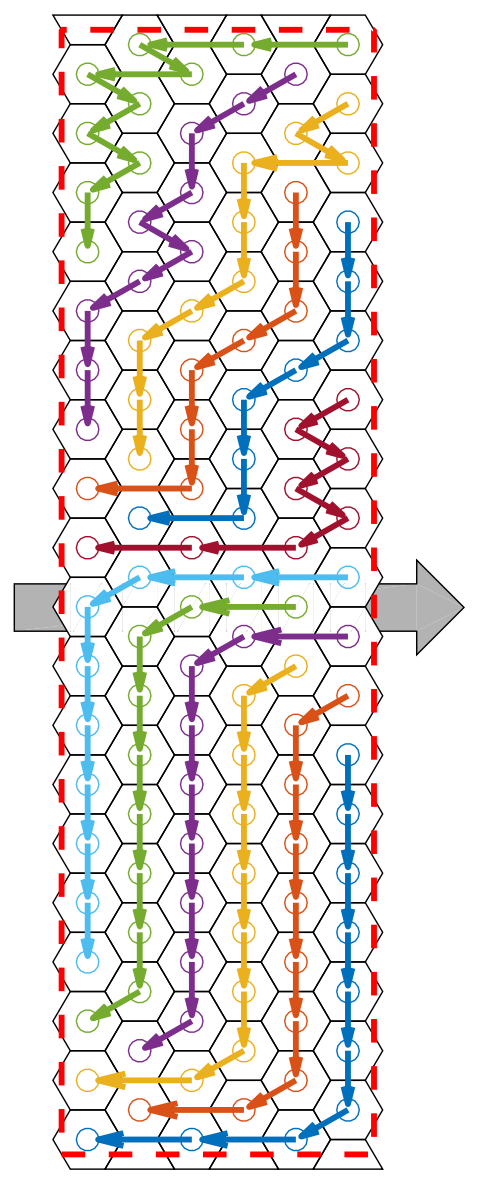

Fig. 1. Side view of the heat exchanger under consideration. The air is passing through the heat exchanger from left to right. The water stream is perpendicular to the shown plane. The connection of the tubes to each other is shown by the small arrows inside the heat exchanger. Each colour refers to one water path. The dashed, red line shows the true boundary of the fins. 
The cell can be as long as one passing tube or only a part of it. Experience with the model shows that the element width has not to be reduced along one tube. To achieve a good fit with the outer boundary of the fins, some truncation or extension via dummy cells can be done. Figure 1 shows the discretisation of one heat exchanger together with the stream paths of the fluids.

\subsubsection{Local model of one cell}

The proposed model is inspired by a model introduced by Wiening [10]. It is modified using a different temperature distribution within one cell. The cell is thought of three sections: The air, the water and the separating tube (see fig. 2). The tube also contains the assigned fins via lumped parameter.

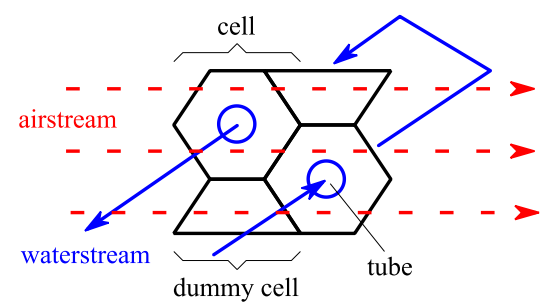

Fig. 2. Scheme of hexagonal cells, which can be divided into a water part (inside the tube), air part (outside the tube) and the separating tube between them.

In the following, the underlying assumptions of the model are described. On the fluid side complete mixing is assumed. This gives a constant air temperature within one cell, which equals the outlet air temperature of this cell. Same can be said for the air humidity and the water temperature. The humid air is assumed to be a mixture of ideal gas, namely water vapour and dry air. In heat transfer the latent and sensible heat of humid air are treated separately. The heat transfer between the cells happens only through convective heat transfer along the flow direction of the fluids. This means that the heat conduction in the tubes or fins is neglected.

With this, the describing quantities in one cell are the air temperature $\vartheta_{a}$ and air humidity ratio $w_{a}$ on the air side, the tube and fin temperature $\vartheta_{t}$ and the water temperature $\vartheta_{w}$. The humidity ratio of the passing air changes, if condensate is diverted. Let $\dot{w}$ be the change in humidity ratio within one cell, $w_{a, \text { in }}$ and $w_{a, \text { out }}$ the humidity ratios of the incoming and outgoing air, $m_{d a}$ the mass of dry air within one cell, $\dot{m}_{d a}$ the mass stream of dry air through the cell and $\dot{m}_{c}$ the mass stream of the condensate within one cell, then the following mass balance describes the change of the humidity ratio:

$$
m_{d a} \dot{w}_{a}=\dot{m}_{d a}\left(w_{a, \text { in }}-w_{a, o u t}\right)-\dot{m}_{c} .
$$

Usually the dynamic of the heat exchange on the air side is much faster than on the water side. Therefore, the derivative of the humidity ratio with respect to time is set to zero, i.e. $\dot{w}_{a}=0$. Then the humidity ratio at the outlet can be calculated by (1). The driving potential for the condensate mass stream $\dot{m}_{v}$ is the difference between humidity ratio $w_{a}$ within the cell and the humidity ratio at the tube's surface, $w_{t}$, which is equal to the saturation humidity ratio at tube temperature. For Lewis Numbers close to 1 the heat transfer coefficient and the mass transfer coefficient for air, $\alpha_{a}$ and $\beta_{a}$, are interrelated to each other by the humid specific heat $c_{p, m}$, which itself can be calculated by $c_{p, m}=\left(1+w_{a}\right) c_{p, h a}$ from the specific heat of the humid air $c_{p, h a}$. Introducing a switch $s$, that is 1 if condensation occurs and zero otherwise,

$$
s=\left\{\begin{array}{l}
1, w_{t}<w_{a} \\
0, w_{t} \geq w_{a}
\end{array}\right.
$$

and let $A_{a}$ be the contact area between tube (including fins) and the air, one obtains:

$$
\dot{m}_{c}=\beta_{a} A_{a}\left(w_{a}-w_{t}\right), \beta_{a}=s \frac{\alpha_{a}}{c_{p, m}} .
$$

The energy balance on the air side is formulated for the sensible heat only. Let $\vartheta_{a, \text { in }}$ and $\vartheta_{a, o u t}$ be the temperature of the incoming and outgoing air and by neglecting the dynamics on the air side again one obtains:

$$
0=\dot{m}_{d a} c_{p, h a}\left(\vartheta_{a, \text { out }}-\vartheta_{a, \text { in }}\right)+\alpha_{a} A_{a}\left(\vartheta_{t}-\vartheta_{a}\right)
$$

For the water side the same procedure can be applied. Let $\dot{m}_{w}$ be the mass stream of water through one tube, $m_{w}$ be the mass of water included in one tube, $\vartheta_{w \text {,in }}$ and $\vartheta_{w, \text { out }}$ be the temperatures of the incoming and outgoing water, $c_{w}$ the specific heat of water, $\alpha_{w}$ the heat transfer coefficient of water and $A_{w}$ the contact area between water and tube. The energy balance then yields

$$
\begin{gathered}
m_{w} c_{w} \dot{\vartheta}_{w}=\dot{m}_{w} c_{w}\left(\vartheta_{w, \text { in }}-\vartheta_{w, \text { out }}\right) \ldots \\
+\alpha_{w} A_{w}\left(\vartheta_{t}-\vartheta_{w}\right) .
\end{gathered}
$$

Between air and water lies the tube. This means that the energy balance for the tube element contains all terms from eqn. (2-5) with opposite sign. Let $m_{t}$ be the mass of the tube (and fins), $c_{t}$ be their average specific heat and $h_{c}$ be the specific enthalpy necessary to evaporate liquid water at the tubes temperature $\vartheta_{t}$ to water vapour at the air's temperature. Altogether this gives

$$
\begin{aligned}
& m_{t} c_{t} \dot{\vartheta}_{t}=\alpha_{a} A_{a}\left(\vartheta_{a}-\vartheta_{t}\right) \ldots \\
& +\alpha_{w} A_{w}\left(\vartheta_{w}-\vartheta_{t}\right)+h_{c} \dot{m}_{c} .
\end{aligned}
$$

The differential equations (5) and (6) describes the rate of change for two state variables in each cell. The other equations allow to calculate the cells output directly.

It should be stated here, that most coefficients are not constant. They are temperature dependent and have to be recalculated from time to time. In the program this is realised by using the temperatures from the previous iteration, assuming only small changes in the values from one time step to another.

\subsubsection{Global model of the overall heat exchanger}

Taking all cells in the presented example together, one gets $n=216$ state space variables. The cells are linked to 
each other through the convective mass streams within the heat exchanger. At the inlet of the exchanger each cell has the same (global) inlet parameters. At the (global) outlet, the outlet values of the last cells are averaged, weighted by the corresponding mass stream.

A signal from the inlet to the outlet needs an amount of time, defined by the fluid's velocity and length of the path. In the simulation this time equals the time step size of the integrator, multiplied by the number of consecutive cells. This gives an upper bound for the time step size of the integrator.

\subsubsection{Linearization and model reduction}

By using the DERIVESTSuite [11] the nonlinear model is numerically linearized. The operating points are chosen in a way that a broad range of inlet variables is covered. Let $\boldsymbol{u}$ and $\boldsymbol{y}$ be the vector of the inlet and outlet variables and $\boldsymbol{x}$ the vector of the states, then the linearized equations become:

$$
\begin{gathered}
\boldsymbol{u}^{T}, \boldsymbol{y}^{T}=\left(\vartheta_{a}, w_{a}, \vartheta_{w}, \dot{m}_{a}, \dot{m}_{w}\right)_{i n, o u t} \\
\dot{\boldsymbol{x}}=A_{i} \boldsymbol{x}+B_{i} \boldsymbol{u}+\boldsymbol{a}_{i}, \boldsymbol{a}_{i}=-A_{i} \boldsymbol{x}_{0 i}-B_{i} \boldsymbol{u}_{0 i}, \\
\boldsymbol{y}=C_{i} \boldsymbol{x}+D_{i} \boldsymbol{u}+\boldsymbol{b}_{i}, \boldsymbol{b}_{i}=\boldsymbol{y}_{0 i}-C_{i} \boldsymbol{x}_{0 i}-D_{i} \boldsymbol{u}_{0 i},
\end{gathered}
$$

where the index $i$ denotes the number of the operating point and the index 0 denotes the value at the operating point.

Since the dimension of the state vector is 216 , this system is less suitable for control design. This can be overcome by model reduction. The idea is, to find a proper transformation $V_{i}$ of the state space variables to reduce the order and to find an additional matrix $W_{i}$ to remove the residuum in the state space equation (the index $r i$ denotes the reduced variable):

$$
\begin{gathered}
W_{i}^{T} V_{i} \dot{\boldsymbol{x}}_{\mathrm{ri}}=W_{i}^{T} A_{i} V_{i} \boldsymbol{x}_{r i}+W_{i}^{T} B_{i} \boldsymbol{u}+W_{i}^{T} \boldsymbol{a}_{i}, \\
\boldsymbol{y}=C_{i} V_{i} \boldsymbol{x}_{r}+D_{i} \boldsymbol{u}+\boldsymbol{b}_{i} .
\end{gathered}
$$

The reduction is performed by the Arnoldi iteration $[12,13]$, which uses the stabilized Gram-Schmidt process. This ensures an orthogonal and normalized reduced base. Typically, the number of the state variables $\check{n}$ can be reduced to be lower than 5 .

If one uses more than one linearization for $\bar{n}$ different operating points, the reduced systems are not necessarily compatible. This is due to the different matrixes $W_{i}$ and $V_{i}$ for each operating point, which results in different reduced states $V_{i} \boldsymbol{x}$. To overcome this, an additional transformation is introduced $[14,15]$. This time the transformation matrix $R$ is the same for each linear model ( $\boldsymbol{x}_{r}$ is the common state variable):

$$
\boldsymbol{x}=V_{i} \boldsymbol{x}_{r i}=R \boldsymbol{x}_{r}, \quad \boldsymbol{x}_{r i}=\left(R^{T} V_{i}\right)^{-1} \boldsymbol{x}_{r} .
$$

The matrix $R$ is chosen in a way that the most important parts of the operating points are considered. Therefore, it is calculated using the singular value decomposition (taking only the first $\hat{n}$ columns and skipping the superfluous columns of $U$ ):

$$
\left[V_{1}, V_{2}, \ldots, \mathrm{V}_{\overline{\mathrm{n}}}\right]=U \Sigma N^{*}, U=[R, \ldots], R \in \mathbb{R}^{n \times \hat{n}} .
$$

Like before, the state space equation is multiplied by a matrix $M_{i}=\left(W_{i}^{T} R\right)^{-1}$ to let the residuum vanish. In practice one should try different combinations for the number of reduced and unified states, to get the best result.

\subsection{Overall system model}

The linearized and reduced model is extended to the air bypass (2.2.1). Albeit the heat exchanger model is linear, the extension gives a nonlinear model and again linearization is necessary (2.2.2).

\subsubsection{Model extension to the air bypass}

The air bypass is controlled via a flap, which allows to control the amount of air separated from the heat exchanger. In general, the air mass stream (total amount and the partition between the bypass and the heat exchanger) is a nonlinear function in the flap position. For the sake of simplicity, we assume in this model a constant total air mass stream and a linear dependence. Let $\varphi$ be the flap position, $\dot{m}_{b}$ and $\dot{m}_{a}$ be the air mass streams through the bypass and the heat exchanger. Both sums up to the total mass stream $\dot{m}_{\text {tot }}$. The splitting and mixing rule can be written as follows:

$$
\begin{gathered}
\dot{m}_{a}=(1-\varphi) \dot{m}_{t o t}, \dot{m}_{b}=\varphi \dot{m}_{t o t}, 0 \leq \varphi \leq 1, \\
\vartheta_{\text {mix }}=\vartheta_{a, \text { out }}(1-\varphi)+\vartheta_{a, \text { in }} \varphi, \\
w_{\text {mix }}=w_{a, \text { out }}(1-\varphi)+w_{a, \text { in }} \varphi .
\end{gathered}
$$

\subsubsection{Linearized overall model}

The linearization and reduction procedure is similar to the scheme introduced before (2.1.4). On the inlet variables the mass stream of the air $\dot{m}_{a, i n}$ is exchanged via the flap position $\varphi$. On the outlet variables, only the air condition $\left(\vartheta_{a}, w_{a}\right)$ is of interest and therefore controlled. For further examination the state vector can be assumed to be of dimension 2 .

\section{Concept of the air bypass}

The air bypass allows to reach for the reference point without reheating. Furthermore, only a part of the air mass passes through the heat exchanger. The outlet temperature from the heat exchanger can therefore be lower than under standard loading conditions. Furthermore, the dynamic of the flap is faster than that of the heat exchanger itself. This can be advantageous in reacting on changing loads in the conditioned room or on changing outdoor air conditions.

A drawback of the bypass is its limited applicability, restricted to cases when the outdoor air is warmer and more humid than the reference point. But if the humidity is too high, the mixing point lies below the reference point and reheating is required. 
For controllability, one has to prove that the reference point lies within the systems capabillities. By using the flap position as one variable, the condtioned air lies on one line, see the pschrometric chart in fig. 3. One can expand this to an area by manipulating the inlet water temperature (mixing the inlet and outlet water streams, see fig. 3, upper chart) or by restricting the inlet water mass stream (see fig. 3, lower chart). Varying the inlet temperature is the more advantegous solution. This is because the curves are more evenly spaced and the rising inlet water temperature allows to weaken the demands on the cold production.
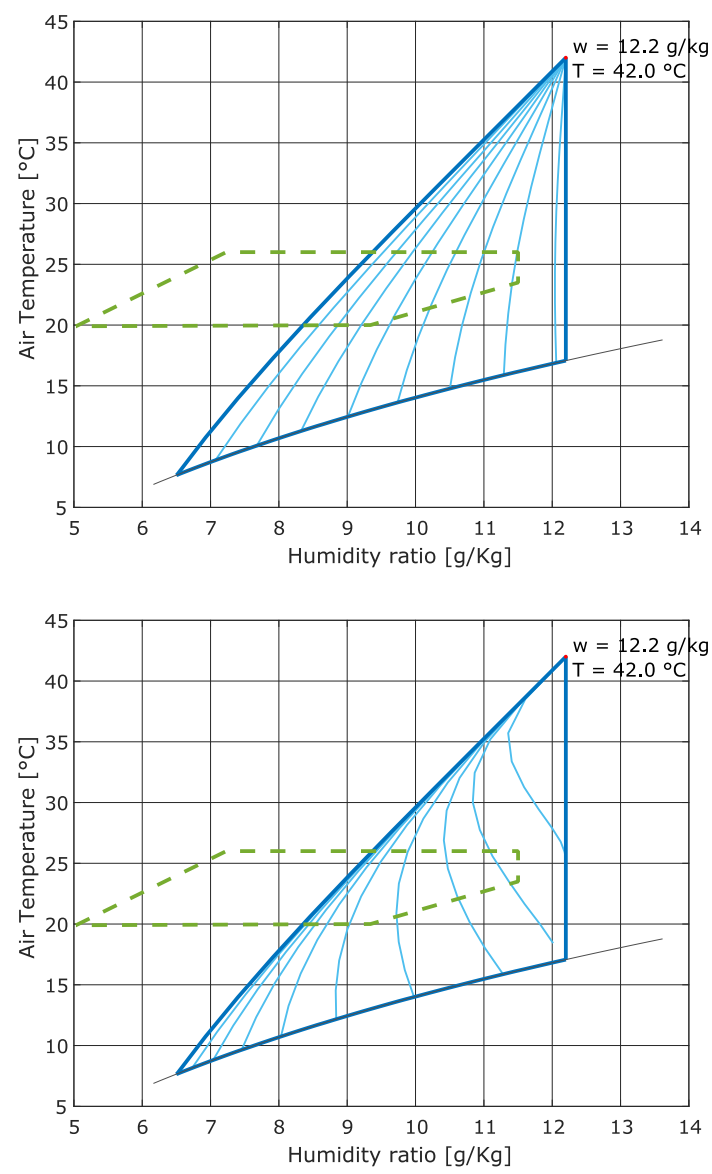

Fig. 3. Psychrometric charts of the reachable area of the conditioned air (famed by the bold, blue line). The comfort area for the conditioned air is shown by the dashed, green line. In the upper right corner is the starting point of the outdoor air. The lower boundary is given by the saturation curve (gray). Along the thin blue lines, the flap position is changed. Different curves are obtained by changing the cooling water inlet temperatures (upper chart) or by changing the cooling water mass stream (lower chart).

\section{Concept of the controller}

For control design, one has to separate the actuating variables from the disturbance variables. As discussed in 3 , the flap position and the inlet water temperature are suitable actuation variables. Let $\boldsymbol{u}_{1}$ and $\boldsymbol{u}_{2}$ be the vectors of the actuating and disturbance variables respectively, then the linear system in one operating points reads as:

$$
\begin{gathered}
\dot{\boldsymbol{x}}_{r}=A_{r i} \boldsymbol{x}_{\boldsymbol{r}}+B_{r i 1} \boldsymbol{u}_{1}+B_{r i 2} \boldsymbol{u}_{2}+\boldsymbol{a}_{r i}, \\
\boldsymbol{y}=C_{r i} \boldsymbol{x}_{\boldsymbol{r}}+D_{r i 1} \boldsymbol{u}_{1}+D_{r i 2} \boldsymbol{u}_{2}+\boldsymbol{b}_{r i}, \\
\boldsymbol{u}_{1}^{T}=\left(\vartheta_{w, i n}, \varphi\right), \boldsymbol{u}_{2}^{T}=\left(\vartheta_{a, i n}, w_{a, i n}, \dot{m}_{w, i n}\right) .
\end{gathered}
$$

As a first draft a linear-quadratic state space controller $[16,17]$ is designed: $\boldsymbol{u}_{1}=-K \boldsymbol{x}$. In the following the matrixes $B_{r i 2}$ and $D_{r i 2}$ are neglected (they will be treated later separately) and $\boldsymbol{y}$ shall consist only of the controlled variables, which describes the air condition at the outlet $\left(\vartheta_{a, \text { mix }}, w_{a, \text { mix }}\right)$. Let $\boldsymbol{y}_{\text {ref }}$ be the vector of the reference variable, then one thinkable cost functional is:

$$
J=\int_{0}^{\infty}\left[\left(\boldsymbol{y}-\boldsymbol{y}_{\text {ref }}\right)^{T} Q_{y}\left(\boldsymbol{y}-\boldsymbol{y}_{\text {ref }}\right)+\boldsymbol{u}_{1}^{T} R_{u} \boldsymbol{u}_{1}\right] d t .
$$

This functional can be reformulated under the assumptions that $\boldsymbol{y}_{r e f}$ is determined by a linear system equation:

$$
\boldsymbol{y}_{r e f}=C_{r e f} \boldsymbol{x}_{r e f}, \dot{\boldsymbol{x}}_{r e f}=A_{r e f} \boldsymbol{x}_{r e f} .
$$

Or in case of a constant value in more simple form:

$$
C_{\text {ref }}=\left(\begin{array}{ll}
1 & 0 \\
0 & 1
\end{array}\right), A_{\text {ref }}=0 .
$$

With the substitution

$$
\widetilde{\boldsymbol{u}}=\boldsymbol{u}+\tilde{R}^{-1} \tilde{S}^{T} \widetilde{\boldsymbol{x}}, \tilde{\boldsymbol{x}}=\left[\begin{array}{c}
\boldsymbol{x} \\
\boldsymbol{x}_{\text {ref }}
\end{array}\right],
$$

and the matrixes

$$
\begin{gathered}
\tilde{Q}=\left[\begin{array}{cc}
Q_{y} & -C_{w}^{T} Q_{y} C_{r i} \\
-C_{w}^{T} Q_{y} C_{r i} & C_{w}^{T} Q_{y} C_{w}
\end{array}\right], \\
\tilde{S}=\left[\begin{array}{c}
C_{r i}^{T} Q_{y} D_{r i 1} \\
-2 C_{w}^{T} Q_{y} D_{r i 1}
\end{array}\right], \\
\tilde{R}=\left[D_{r i 1}^{T} Q_{y} D_{r i 1}+R_{u}\right] .
\end{gathered}
$$

the functional (20) can be reformulated

$$
J=\int_{0}^{\infty}\left[\widetilde{\boldsymbol{x}}^{T} \tilde{Q} \widetilde{\boldsymbol{x}}+2 \widetilde{\boldsymbol{x}}^{T} \tilde{S} \widetilde{\boldsymbol{u}}+\widetilde{\boldsymbol{u}}^{T} \widetilde{R} \widetilde{\boldsymbol{u}}\right] d t
$$

Insert a state space controller $\widetilde{\boldsymbol{u}}=-\widetilde{K} \widetilde{\boldsymbol{x}}$ and minimizing the cost functional gives $\widetilde{K}$. From (23) then the sought after controller $K$ is obtained.

The choice of the matrixes $Q_{y}$ and $R_{u}$ is crucial. Without any further restrictions they contain 8 parameters, which have to be varied over many magnitudes to find an optimal controller, which doesn't has too severe demands on the actuating variables. By way of example the inlet water temperature cannot be below the temperature of water supplied by the cold production, usually 6 degrees Celsius.

This procedure can be done for every operating point separately. By a gain scheduling scheme $[18,19]$ the overall controller is found. The scheduling is performed by the state space variable $\boldsymbol{x}$ of dimension 2 . The membership function is composed of piecewise trapezoid functions. It should be mentioned that the combined controller cannot guaranteed to be stable, since the design is local only. 
In future works, an all-embracing controller design is sought. In this, a linear matrix inequalities (LMIs) are derived from a Lyapunov function [20]. By adding parameters to the Lyapunov function the decay rate and the dynamic of the controlled system can be influenced. Advantageous is the stability, which can be guaranteed for the linear system in one operating point as well as for the superposed system. Furthermore, the experimental validation of the controller is left over for future work. A complete test facility is available for this.

\section{Conclusions}

A very general modelling concept for heat exchangers is described. This is, by means of the easy adoptable local system of differential equations, derived from energy balance equations and the linking of the two different mass streams. Disadvantageous of this model is the nonlinear nature of the describing equations and the large number of states. To overcome the first, a numerical linearization procedure is applied for different operating points. The second one is addressed by application of a reduction method. Care has to be taken, since the different reduced models are not compatible to each other. This problem is resolved in a second step by using the singular value decomposition to find a proper second linear reduction transformation.

Furthermore, the bypass concept to spare the reheating of cooled (and dehumidified) air is introduced and discussed. It can be advantageous by means of energy consumption due to avoidance of reheating the conditioned air. Further studies have to be done to examine its potential, depending on the usual weather conditions and individual demands within conditioned rooms. The mechanical expansion of existing hvac units by an air bypass is relatively simple. This means the concept can be applied to existing facilities as well as to new ones.

A first draft of the controller is given. The linearquadratic controller gives the optimal control matrix by means of a cost functional. This design can be done for each operation point separately. The overall controller is obtained through a gain scheduling scheme. Disadvantage of this method is the not guaranteed stability for the overall controller. To overcome this, later designs will rely on a Lyapunov function which takes into account each model at the same time. The resulting LMIs can be solved by already established convex optimization algorithm.

This research project is funded by the Senate Department for Urban Development and Environment, Berlin, Germany under grant no. 1050-B5-O.

\section{References}

1. N. B. Grimm, S. H. Faeth, N. E. Golubiewski, C. L. Redman, J. Wu, X. Bai, J. M. Briggs, Science, 319, 5864, 756-760 (2008)

2. P. Sheng, X. Guo, Energy, 165, B, 118-125 (2018)

3. L. Prez-Lombard, J. Ortiz, C. Pout, Energy and Buildings, 40, 394-398, 01 (2008)

4. P. Krimmel, N. Goldschmidt, R. Meier, H. Schulte, B. Müller, CLIMA 2016 (2016)

5. N. Goldschmidt, H. Schulte, IFAC-PapersOnLine, 50, 1 (2017)

6. D. Schwingshackl, J. Rehrl, M. Horn, Building and Environment, 96, 250-259 (2016)

7. D. Schwingshackl, J. Rehrl, M. Horn, J. Belz, O. Nelles, Journal of Building Engineering, 11, 224-229 (2017)

8. S. Kabelac, International Journal of Heat and Mass Transfer, 32, 6, 1183-1189 (1989)

9. K. R. McCullagh, G. H. Green, S. Chandrasekar, ASHRAE Transactions, 75, II, 200-209 (1969)

10. W. Wiening, Fortschritts-Berichte VDI, 128 (1987)

11. J. D'Errico, Adaptive Robust Numerical Diff., https://de.mathworks.com/matlabcentral (2014)

12. W. E. Arnoldi, Quarterly of Applied mathematics, 9, 17-29 (1951)

13. G. H. Golub, C. F. Van Loan, Matrix Computations (1996)

14. B. Salimbahrami, B. Lohmann, T. Bechtold, J. G. Korvink, Mathematical and Computer Modelling of Dynamical Systems, 11, 1, 79-93 (2005)

15. H. Panzer, J. Mohring, R. Eid, B. Lohmann, Automatisierungstechnik, 58, 8, 958 (2010)

16. H. Kwakernaak, R. Sivan, Linear Optimal Control Systems (1972)

17. E. Sontag, Mathematical Control Theory: Deterministic Finite Dimensional Systems (1998)

18. D. J. Leith, W. E. Leithead, International Journal of Control, 73, 11, 1001-1025 (2000)

19. D. A. Lawrence, W. J. Rugh, Automatica, 31, 3, 381390 (1995)

20. H. K. Khalil, Nonlinear Systems (1996) 